\title{
Polymer based nano-assemblies: very efficient carrier in the field of cancer chemotherapy
}

\begin{abstract}
Self-assembly of amphiphilic polymer opens a new avenue in the biomedical field; particularly, amphiphilic polymer vesicles have generally shown promising potential in drug delivery applications. The advantage of nanoparticle for drug delivery is because of the direct injection of these kind molecules to the body which will circulate in the body without any coagulation or blocking. The amphiphilic nature makes the micelles an effective carrier for the drugs, which are struggling with poor solubility, limited stability, and toxicity.
\end{abstract}

Volume 5 Issue 6 - 2017

\author{
Saikat Mukherjee, Raja Shunmugam \\ Department of Chemical Sciences, Indian Institute of Science \\ Education and Research Kolkata, India
}

Correspondence: Raja Shunmugam, Polymer Research Centre, Department of Chemical Sciences, Indian Institute of Science Education and Research Kolkata, Mohanpur- 741246, India, Emailsraja@iiserkol.ac.in

Received: July 01, 2017 | Published: July 13, 2017

\section{Introduction}

Cancer is a disease that causes for millions of deaths throughout the world every year. ${ }^{1}$ Different decisive steps have already been made but most of the cellular processes causes for cancer is either unknown or not been translated into the effective cure. ${ }^{2}$ Among different mode of treatments (e.g. radiotherapy, immunotherapy, and chemotherapy) chemotherapy with its different organic molecules or inorganic metals, is the central component of the treatment. ${ }^{2}$ However, small molecule drugs (e.g. doxorubicin, camptothecin, cis-platin) suffer either from solubility or site-specificity causes for severe sideeffects in real applications. ${ }^{3}$ To deal with these different drawbacks of small molecule drugs, researchers have employed different delivery vehicle carrying the chemotherapeutic agent either with non-covalent encapsulation or attached with different stimuli responsive $(\mathrm{pH}$, thermal, and enzymatic) linkers to the delivery vehicle ${ }^{3-13}$. This vehicle carries single or multi drug component in aqueous environment form self-assembled nano-structure by protecting the drugs into its hydrophobic core and deliver the drug once it reaches to the affected area. $^{3-13}$ There are several systems (e.g. nanoparticles, peptides, dendrimer, and liposomes) have been explored by the researchers which efficiently carries the drug to the targeted sites. ${ }^{4}$ These all particularly form excellent nano-assemblies in aqueous environment due its amphiphilicity present in it. ${ }^{14-18}$ Polymer based drug delivery system is one of those which form excellent nano-assemblies when it functionalized with hydrophilic PEG group. ${ }^{7,9,11-13}$ The drug can deliver through this nano-carrier either by non-covalent encapsulation or covalently attached with different stimuli responsive linkers. ${ }^{5,7,13-15}$ The polymer based nano-vehicle shows enhanced cytotoxic effect due to its plasma stability which eventually helps to increase the permeation and retention effect $(\mathrm{EPR})^{5-15}$ Due to leaky vesicular nature of the blood vessel around the tumour help these nano-vehicles to reach the targeted sites. ${ }^{8}$ In this modern era, these delivery vehicles are even making more site-specific by incorporating different pendent receptor moieties into it. ${ }^{9}$ Due to abnormal growth of the tumours it uptake more amount of nutrients, mineral, and vitamins compare to normal cells which causes the formation of overexpressed receptors onto the cancer cell surface. Folic acid and Vitamin H (biotin) are one of those whose receptors are found overexpressed onto cancer cellular surface. ${ }^{3}$ Hence, functionalization of these site-specific motifs into the nano-assemblies, which targets the receptors those are over expressed on the surface of the cancer cell, make the system more sitespecific. Further, these nano-assemblies are internalized efficiently by following receptor mediated endocytosis, a well-known mechanism followed by mammalian cells (Figure 2) ${ }^{7,9,13}$


Figure I Self-assembled nano-aggregate of amphiphilic polymer with pendent receptor motif in aqueous condition.



Figure 2 A schematic representation of site-specific delivery of polymeric nano-assembly.

Different polymeric methods with different backbones (e.g. ROP, poly-condensation, ROMP), have already been explored by different research groups to employ various functionalities for synthesizing smart carriers in the field of nano-medicine. ${ }^{5-14}$ These different polymeric system shows excellent nano-assembly. ${ }^{5-14}$ Upon incorporation of drugs into that by either covalent or non-covalently form different shapes at nano-scale level and internalize inside the cell 
quite efficiently. ${ }^{5-15}$ Drugs attached through different stimuli responsive covalent linkers follow sustain release mechanism, provide therapeutic response over longer time period. ${ }^{5,7,9,11-13}$ Whereas non-covalently encapsulated drug follows the burst mechanism, gives a sudden killing of cancer cell at post-metastatic stage. ${ }^{15}$ So, a multifunctional nano-assembly carries the drug with generally considered as nanomedicine is due to its ability to form self-assembled structure at the nano-meter level..$^{5-15}$ This nano-medicine open up a new way to fine tune the properties by simply altering the functional motifs attached to the carriers and gives a better therapeutic efficacy in terms of sitespecificity and solubility. ${ }^{4-15}$

Recently, drug delivery nano-vehicles are gearing up towards the zone of theranostics, a system composed of therapeutic and diagnostic agent together. ${ }^{7,913}$ A synthetic architectural molecule functionalized with different imaging probes (MRI contrast agent, Fluorescent agent, and NIR-imaging probe) along with the therapeutic agent open up a new avenue of treating malignancies. ${ }^{7,9,13,16}$ This modified system can diagnose the affected area along with treatment by carrying the both in a single system and this actually help to monitor the therapeutic pathway of the drug. ${ }^{7}$ These systems also help to monitor the therapeutic efficacy of the non-emissive drugs (chlorambucil, cisplatin) in each dose. ${ }^{5,9,12}$ So, to incorporate the different functionalities into a single polymeric system there is always a pressing need to have a living polymerization technique which can form a range of different molecular weight polymer with uniform distribution. ${ }^{7,13}$ Ring opening metathesis polymerization (ROMP) is one of those technique, where a highly functional group tolerant Grubbs' catalyst gives the opportunity to make excellent polymers, bearing different functionalities with narrow poly dispersity. ${ }^{7}$ Grubbs' catalyst with its three different generation ruthenium catalysts can open the norbornene ring with different complex architecture which eventually help to tune the different properties of the biocompatible nano-carrier. ${ }^{13}$ Theranostic systems or site specific dual imaging motifs generally requires of anchoring different complex synthetic molecules into a single system to tune the properties and metathesis polymerization technique serve it excellently.,17,19 By this method different nano-mater aggregated structure can be formed with different imaging motif and drug attached to it by variety of stimuli responsive linker or encapsulation. ${ }^{7,15,17}$ The functional group tolerant Grubbs' catalyst can polymerize a variety of monomers bearing complex motif or inorganic metal and thus we can tune the loading by simply fine tuning the degree of polymerization (DP). ${ }^{13,17}$

In summary, Polymer based nano-assembled aggregates are promising tool for the purpose of delivery of drug or theranostic molecules. Incorporation of folic acid or biotin into the polymeric system makes the system even more site-specific. This long chain ampiphilic molecule form small core-shell nano-aggregate by keeping the hydrophobic drug or imaging motifs into its core and peg with its receptor molecule as pendent motif. The different stimuli responsive covalent linkers can be used for sustained release of the drug whereas for burst release mechanism non-covalent encapsulation is required.

\section{Future prospect}

Recent reports show that the functional group tolerant Grubbs' catalyst can form the different range of cyclo-poly-acetylene with uniform distribution and different functionality can be employed to that to regulate its property in terms of solubility, and so on. ${ }^{20}$ This biocompatible molecule can open up a new avenue to make complex architectural molecule with better response in the field of cancer therapy. ${ }^{20}$

\section{Conflicts of interest}

None.

\section{Acknowledgments}

S.M. thanks CSIR, New Delhi, for their research fellowships. R.S. thanks the Department of Science and Technology, New Delhi, for the Ramanujan Fellowship and DST-SERB (EMR/2015/001600) for funding. R.S. thank IISER-Kolkata for supplying infrastructure and start-up funding.

\section{References}

1. Siegel RL, Miller KD, Jemal A. Cancer Statistics, 2016. CA: Cancer J Clin. 2016;66(1):7-30.

2. Miller KD, Siegel RL, Lin CC, et al. Cancer Treatment and Survivorship Statistics, 2016. CA Cancer J Clin. 2016;66(4):271-289.

3. Hurley LH. DNA and its processes as targets for cancer therapy. Nat Rev Cancer. 2002;2:188-200.

4. Kapri S, Maiti S, Bhattacharyya S. Lemon Grass Derived Porous Carbon Nanospheres Functionalized for Controlled and Targeted Drug Delivery. Carbon. 2016;100:223-235.

5. Saha B, Haldar U, De P. Polymer-Chlorambucil Drug Conjugates: A Dynamic Platform of Anticancer Drug Delivery. Macromol Rapid Commun. 2016;37:1015-1020.

6. Pramanik P, Halder D, Jana SS, et al. pH-Triggered Sustained Drug Delivery from a Polymer Micelle having the $\beta$-Thiopropionate Linkage. Macromol Rapid Commun. 2016;37(18):1499-1506.

7. Mukherjee S, Dinda H, Chakraborty I, et al. Engineering CamptothecinDerived Norbornene Polymers for Theranostic Application. ACS Omega. 2017;2(6):2848-2857.

8. Wehrung D, Chamsaz EA, Andrews JH, et al. Engineering Alkoxyphenacyl-Polycarbonate Nanoparticles for Potential Application in Near-Infrared Light-Modulated Drug Delivery via Photon UpConversion Process. J Nanosci Nanotechnol. 2017;17:4867-4881.

9. Mukherjee S, Sarma JD, Shunmugam R. pH-sensitive nano aggregates for site-specific drug-delivery as well as cancer cell imaging. ACS Omega. 2016; 1(5):755-764.

10. Oz Y, Arslan M, Gevrek TN, et al. Modular Fabrication of Polymer Brush Coated Magnetic Nanoparticles: Engineering the Interface for Targeted Cellular Imaging. ACS Appl Mater Interfaces. 2016;8(30):19813-19826.

11. Bhattacharya S, Ganivada MN, Dinda H, et al. Biodegradable Copolymer for Stimuli-Responsive Sustained Release of Doxorubicin. ACS Omega. 2016;1:108-117.

12. Deshpande NU, Jayakannan M. Cisplatin-Stitched Polysaccharide Vesicles for Synergistic Cancer Therapy of Triple Antagonistic Drugs. Biomacromolecules. 2017;18(1):113-126.

13. Mukherjee S, Patra D, Dinda H, et al. Super paramagnetic norbornene copolymer functionalized with biotin and doxorubicin: A potential unique site-Specific theranostic agent. Macromolecules. 2016;49(7):2411-2418.

14. Mane SR, Sathyan A, Shunmugam R. Synthesis of Norbornene Derived Helical Copolymer by Simple Molecular Marriage Approach to Produce Smart Nanocarrier. Sci Rep. 2017;7:44857.

15. Mane SR, Rao VN, Chaterjee K, et al. Amphiphilic Homopolymer Vesicles as Unique Nano-Carriers for Cancer Therapy. Macromolecules. 2012;45:8037-8042.

16. Glassner M, Palmieri L, Monnery BD, et al. The Label Matters: $\mu$ PET Imaging of the Biodistribution of Low Molar Mass 89Zr and 18FLabeled Poly(2-ethyl-2-oxazoline). Biomacromolecules. 2017;18:96-102. 
17. Mukherjee S, Shashank L, Dinda H, et al. Site-specific amphiphilic magnetic copolymer nano-aggregates for dual imaging. Macromolecules. 2015;48 (19):6791-6800.

18. Banerjee S, Maji T, Mandal TK. Graft copolymers via combination of cationic polymerization and atom transfer radical polymerization and their phase separation into spherical/worm-like nanostructures. Colloid Polym Sci. 2014;292(9):2217-2226.
19. Backlund CM, Sgolastra F, Otter R, et al. Increased hydrophobic block length of PTDMs promotes protein internalization. Polym Chem. 2016;7(48):7514.

20. Ganivada MN, Kumar P, Babu A, et al. Engineering a New Class of Multiarm Homopolymer for Sustainable Drug Delivery. ACS Biomater Sci Eng. 2017;3(6):903-908. 